\begin{tabular}{|c|c|c|c|c|c|c|}
\hline & Sensitivity & Specificity & Youden & $\begin{array}{c}\text { AUC } \\
(\mathrm{CI} 95 \%)\end{array}$ & $\begin{array}{c}\text { OR } \\
(\mathrm{CI} 95 \%)\end{array}$ & AIC \\
\hline Low C3 $(<81 \mathrm{mg} / \mathrm{dl})$ & $27 \%$ & $97 \%$ & 0.240 & $\begin{array}{c}0.620 \\
(0.599,0.641)\end{array}$ & $\begin{array}{c}10.9 \\
(6.28,18.91)\end{array}$ & 1200 \\
\hline Low C4 (<12.9 mg/dl) & $27 \%$ & $97 \%$ & 0.235 & $\begin{array}{c}0.618 \\
(0.596,0.639)\end{array}$ & $\begin{array}{c}9.57 \\
(5.67,16.15)\end{array}$ & 1206 \\
\hline Low C3 or low C4 & $38 \%$ & $93 \%$ & 0.313 & $\begin{array}{c}0.656 \\
(0.632,0.681)\end{array}$ & $\begin{array}{c}8.34 \\
(5.55,12.53)\end{array}$ & 1174 \\
\hline Abnormal EC4d (>14 net MFI) & $43 \%$ & $92 \%$ & 0.350 & $\begin{array}{c}0.675 \\
(0.650,0.700)\end{array}$ & $\begin{array}{c}8.67 \\
(5.9,12.72)\end{array}$ & 1153 \\
\hline Abnormal BC4d ( $>60$ net MFI) & $50 \%$ & $94 \%$ & 0.436 & $\begin{array}{c}0.718 \\
(0.693,0.743)\end{array}$ & $\begin{array}{c}14.95 \\
(9.81,22.77)\end{array}$ & 1082 \\
\hline Abnormal EC4d or BC4d & $62 \%$ & $88 \%$ & 0.492 & $\begin{array}{c}0.746 \\
(0.719,0.772)\end{array}$ & $\begin{array}{c}11.05 \\
(7.95,15.37)\end{array}$ & 1067 \\
\hline
\end{tabular}

Methods All subjects $(\mathrm{n}=1200)$ were adults $(18$ years $)$ and enrolled from multiple academic Centers in the United States. All SLE fulfilled the 1997 ACR criteria for SLE $(n=498)$. Patients with Other Rheumatic Diseases $(n=450)$ consisted of 189 rheumatoid arthritis, 88 Sjogrens, 90 Fibromyalgia and 83 patients with other connective tissues diseases. A group of healthy normal individuals was also enrolled $(n=252)$. Abnormal CB-CAPs status (EC4d or BC4d $>99$ th percentile of normal) were determined using flow-cytometry. Complement C3 and C4 levels were determined using immunoturbidimetry (Binding Site, San Diego, CA) assay kits. Performances of the markers, either alone or in combination to distinguish SLE from other rheumatic diseases and controls were established using Sensitivity, Specificity, Odds Ratio and Area under the Curve (AUC) of the Receiver Operating Characteristic curve (ROC). Youden Index (Sensitivity + Specificity - 100) was also calculated. The combination of 4 complement marker abnormalities were also evaluated using logistic regression and unweighted composite score cumulating the presence of these abnormalities was calculated.

Results Abnormal CB-CAPs status yielded 62\% sensitivity with $88 \%$ specificity in distinguishing SLE from the group of patients with other diseases (table 1). Youden Index was 0.492 \pm 0.027 . Low C3/C4 status yielded $38 \%$ sensitivity and $93 \%$ specificity in distinguishing SLE from the group of patients with other diseases. Youden Index was $0.313 \pm 0.025$ for Low $\mathrm{C} 3$ or $\mathrm{C} 4$ and significantly lower than Youden score associated with abnormal CB-CAPs $(p<0.01)$. Specificity of Low C3/C4 and abnormal CB-CAPs in distinguishing SLE from normal healthy individuals was $93 \%$ and $99 \%$, respectively. AUC was also significantly higher with $\mathrm{BC} 4 \mathrm{~d}(0.718)$ than with low $\mathrm{C} 3$ (0.620; p <0.01), low C4 $(0.618 ; \mathrm{p}<0.01)$ and Low C3 or C4 status $(0.656 ; \mathrm{p}<0.01)$. A composite score (unweighted) cumulating all 4 abnormalities, was higher in SLE $(1.47 \pm 0.06)$ than disease control group $(0.21 \pm 0.02)(\mathrm{p}<0.01)$ and normal healthy individuals $(0.01 \pm 0.02) \quad(\mathrm{p}<0.01)$. The complement scoring system yielded higher AUC (0.812), higher OR (36.0 CI95\%: 18.8-69.0), lower AIC (1037) and greater R2 (0.403) than any other combinations
Conclusions Our data suggests that CB-CAPs have greater diagnostic yield than low complement $\mathrm{C} 3 / \mathrm{C} 4$. The combination of these complement abnormalities in composite complement score has high yield in distinguishing SLE from other rheumatic diseases and normal healthy individuals.

Funding Source(s): Exagen.

\section{LEUKOCYTE IMMUNOGLOBULIN-LIKE RECEPTOR A3 (LILRA3) PROMOTES LUPUS-LIKE DISEASE IN A MURINE LUPUS MODEL}

Jianping Guo*, Yuxuan Wang, Yundi Tang. Peking University, People's Hospital

\subsection{6/lupus-2019-Ism.238}

Background Leukocyte immunoglobulin-like receptor A3 (LILRA3) is a secreted protein belongs to LILR family. Our research group previously reported that the functional LILRA3 is a novel genetic risk for multiple autoimmune diseases including systemic lupus erythematosus (SLE). However, the function of LILRA3 in development of lupus is unclear. The bm12 model is a chronic graft-versus-host disease (cGVHD) model characterized by the lupus-like syndrome with cell subtype alteration and autoantibody production. To functionally study the role of LILRA3 in lupus pathogenesis, we constructed the LILRA3 knock-in mice and assessed the clinical manifestation and immune responses in bm12 model.

Methods Human LILRA3 gene (Gene ID: 11026) was inserted into Rosa26 allele in C57BL/6 (B6) mice based on Cas9/ sgRNA system. The cGVHD was induced by an intraperitoneal injection of the bm12 donor splenocytes into recipients, either B6 wild-type or knock-in mice. Mice were sacrificed on day 14 and 28. Flow cytometry was used to analyze frequencies of immunocytes from mice splenocytes. Enzyme-linked immunosorbent assay was applied to detect antibodies in serum.

Results Compared with the wild-type mice, the LILRA3 knock-in mice displayed a more severe immune response on 
both day 14 and day 28 of post induction. Spleen mass were significantly increased in LILRA3 knock-in mice $(p<0.001)$. The proportion of Th2, TFH, germinal center (GC) B, and plasma B cells were increased in knock-in mice $(p<0.01)$, but not Th17 and regulatory $\mathrm{T}$ cells. Concentration of serum antidsDNA IgG was significantly elevated in knock-in mice $(\mathrm{p}<0.001)$.

Conclusions Our data indicate that LILRA3 promotes lupuslike disease probably through the excessive expression of Tfh cells and GC B cells, subsequently help for the induction and maintenance of plasma cell differentiation and autoantibody production.

Funding Source(s): None

\section{PATTERNS OF ORGAN INVOLVEMENT IN SLE AND THEIR OUTCOME: A REAL LIFE EXPERIENCE IN A LUPUS CLINIC}

${ }^{1}$ Md Mujibur Rahman*, 'Md Moniruzzan, 'Jakaria Been Sayeed, ${ }^{2}$ Rashedul Hassan, ${ }^{3}$ Satyajit Barua, ${ }^{4}$ Shaoun Barua, ${ }^{1}$ Md Uzzwal Mallik, ${ }^{4}$ Abdur Rahim. 'Dhaka Medical College; ${ }^{2}$ Green Life Medical College, Dhaka; ${ }^{3}$ Shaheed Suhrawardy Medical College Hospital; ${ }^{4}$ Shaheed Suhrawardi Medical College

\subsection{6/lupus-2019-Ism.239}

Background Systemic lupus erythematosus (SLE) is a multisystem autoimmune disorder predominantly affecting the women of childbearing age. It often manifests with various constitutional symptoms as well as combination of organ systems involvement and outcome varies in different population with available treatment. The present study is to see the patterns of organ involvement and their outcomes at first 6 months with standard treatment.

Methods This was a retrospective study done in lupus clinic of Shaheed Suhrawardy Medical College Hospital, Dhaka, Bangladesh during 2010 to 2016. Patients were included based on ACR lupus diagnostic criteria and had received standard treatment. Outcomes were assessed regularly by clinical features, urinalysis and serum creatinine done in the appropriate cases. Composite endpoint was calculated by using SELENA SLEDAI in all the cases.

Results Among 120 patients, 111 (92.5\%) were female, age ranging from 14 to 57 years with a mean of $26.5 \pm 10.68$ (SD) year. Median follow up was 1.7 years. The most common manifestations were fever (70\%), joint pain $(72.5 \%)$, oral ulceration (49\%), alopecia (40\%), malar rash (28.3\%), photosensitivity (25.8\%) and Raynauds phenomenon (19\%). Commonly involved major organ-systems were renal (40\%), neuropsychiatric (19\%), respiratory (14\%), cardiac (6.6\%) and hematological (6.6\%). In lupus nephritis, proteinuria was present in $100 \%$ of cases. The other parameters of renal involvements were $\mathrm{RBC}>5 / \mathrm{HPF}$ and $\mathrm{RBC}$ or cellular cast in $37.5 \%$. Serum creatinine was raised in 23 patients $(19.17 \%)$ with a mean of $1.68 \pm 0.96 \mathrm{mg} / \mathrm{dl}$. Renal biopsy was done in $40(83.3 \%)$ cases. The histology showed class-II in $03(7.5 \%)$, Class-III in $03(7.5 \%)$, class-IV in $20(50 \%)$ and class-V in 08 $(20 \%)$ cases. With standard treatment, major reduction of mean serum creatinine, 24 hours UTP and SLEDAI were observed in most of the cases at least after 6 months. Total flare occurred in $31(25.8 \%)$ among them renal flare was in $10(8.33 \%)$ and non-renal flare in 21 (17.5\%). Four SLE patients died during the course of treatment.
Conclusions Renal and central nervous systems are the most commonly involved major organ systems. The overall outcome is favorable with standard treatment.

Funding Source(s): None

\section{\begin{tabular}{|l|l}
240 & TRANS-ANCESTRAL EXCLUSION MAPPING STRATEGY
\end{tabular} PRIORITISES RISK ALLELES AT IKZF3 WITH INCREASED BIOLOGICAL RELEVANCE}

${ }^{1}$ Deborah S Cunninghame Graham*, SLE Immunochip Consortium, ${ }^{2}$ Timothy Vyse. ${ }^{1} \mathrm{KCL}$; ${ }^{2}$ King's College London

10.1136/lupus-2019-Ism.240

Background Pinpointing causal risk alleles, carried on extended risk haplotypes, at susceptibility loci for common disease is challenging. We present a novel strategy to prioritise tag-SNPs at risk loci with the highest likelihood of biological relevance for laboratory functional studies. This strategy involves transancestral mapping of risk-haplotypes, MAF-exclusion-mapping of discordant variants, followed by co-localisation of tag-SNPs with epigenetic annotation/identification of allele-specific transcription factor (TF) binding sites, using data from public databases such as RoadMap, ENCODE and HaploRegv4, as a surrogate for causality.

We illustrate the utility of this approach at the Ikaros zinc finger TF IKZF3, with a $194 \mathrm{~kb}$ associated European (EUR) SLE haplotype carrying 282 tag-SNPs, extending over multiple genes from the IKZF3 3 flanking-region into the upstreamregion of ORMDL3.

Methods Align IKZF3 control haplotypes from our large EUR GWAS and five 1000G super-populations. Compare EUR-African MAF to delineate discordant tag-SNPs. Use SLE ImmunoChip data to exclude variants exhibiting $>3 \% \mathrm{MAF}$ and not associated $(\mathrm{p}>0.05)$ in African-Americans (AA). Undertake colocalisation analysis, using multiple tools (Coloc-stats webserver), to prioritise risk alleles overlapping DNAse I hotspots and chromatin modifications characterising active enhancers and/or promoters in blood cell-types (RoadMap data). Additional evidence of potential function was sought for chromatin looping (3D Genome Browser) and differential TF binding (HaploRegv4).

Results Trans-ancestral mapping reduced the risk-haplotype by $47 \%$ to $101 \mathrm{~kb}$ and the tag-SNPs by $28 \%$ to 140 . 26 tagSNPs exhibited association in both EUR and AA SLE ImmunoChip cohorts. All 26 variants reside in regions of open chromatin in LCLs. 15 of the tag-SNPs lie within regions involved in chromatin-looping events, bringing together the full-length promoter and I1 of a shorter IKZF3 isoform. We discovered allele-specific binding of Fox family members to risk alleles in the promoter (rs111678394) and variants in I1 of the shorter isoform: rs113730542 and rs112876941.

Conclusions We hypothesise that allele-specific binding of Fox TFs to risk alleles in the promoter and/or I1 of the shorter isoform IKZF3 may independently modulate the expression of both isoforms. Since the shorter isoform lacks the four zinc fingers responsible for DNA binding of Aiolos (E4-6), the shorter isoform is unable to directly bind DNA. Heterodimerisation between the two isoforms may therefore sequester the full-length isoform in a biologically inactive form. If Fox TFs stabilise chromatin loops within IKZF3 in an allele-specific manner, this may provide a mechanism where risk alleles 